\title{
Comparison of the Full-Potential and Euler Formulations for Computing Transonic Airfoil Flows
}

J. Flores, J. Barton, T. Holst, and T. Pulliam

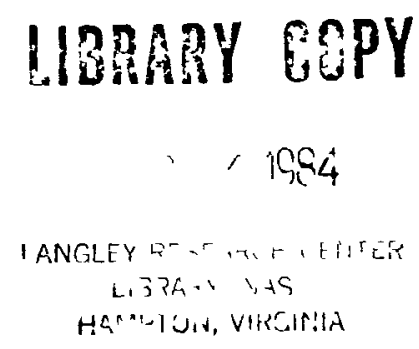




\section{Comparison of the Full-Potential and Euler Formulations for Computing Transonic Airfoil Flows}

J Flores

$J$ Barton

$T$ Holst

T Pullıam, Ames Research Center, Moffett Field, Calıfornıa

\section{N/Sก}

Natıonal Aeronautics and

Space Admınıstratıon 


\author{
J. Flores, J. Barton, T. Holst, and T. Pulliam \\ NASA Ames Research Center \\ Moffett Field, CA 94035 USA
}

\title{
I. INTRODUCTION
}

Recently, much attention has been directed toward developing the Euler formulation for various applications in transonic aerodynamics. However, little effort has been made to compare the speed, accuracy, and robustness of these new Euler codes with the full-potential (FP) formulation. The purpose of this paper is to make such a quantitative comparison using a number of transonic airfoil cases.

The computed results are from four transonic alrfoil computer codes: (1) TAIR $[1,2]$; (2) FLO36 [3]; (3) ARC2D [4,5], and (4) FLO52R [6]. Codes (1) and (2) are FP codes, and codes ( 3 ) and (4) are Euler codes. The FP codes (TAIR and FLO36) use fully 1mplic1t iteration algorithms (AF2 and ADI, respectively); the convergence speed of FL036 is further enhanced by a multigrid convergence acceleration process. The first Euler code (ARC2D) uses a fully implicit ADI iteration scheme; the second (FL052R) uses an explicit Runge-Kutta time-stepping algorithm, which is enhanced by a multigrid convergence acceleration scheme.

The TAIR and ARC2D codes were each run using two types of grids. One grid was generated numerically, using an elliptic (Laplacian) solver [2], and the second was generated from an algebralc routine [7]. The FLO36 and FLO52R codes were run using an internally generated grid of the circle plane mapping variety.

The comments and conclusions reached in this study will be expressed generally, that is, in terms of FP versus Euler. The reader should bear in mind that these conclusions have been reached using the four specific codes mentioned above. We expect the results presented herein to be tvpical, but other codes that use different spatial or iteration algorithms may produce somewhat different results.

\section{RESULTS}

Figure 1 is a plot of lift coefficient versus the average mesh spacing on the alrfa11. The alrfo1l is a NACA 0012, and the flow conditions are $M_{\infty}=0.63$ and $\alpha=2.0^{\circ}$. As the grid was refined, the ratio of the number of grid points along the airfoil to the number of grid points away from the airfoll was held fixed. The outer boundary was placed at 12 chords from the airfoil. A study, which consisted of plotting the $11 \mathrm{ft}$ versus distance to the outer boundary, was conducted; it verified that this distance was sufficient to remove outer boundary effects. The TAIR and FLO36 codes produce 1 ift asymptotes of 0.3326 and 0.3333 , respectively, and the ARC2D and FLO52R codes produce asymptotic values of 0.3357 and 0.3342 , respectively.

Theoretically, all results for the two formulations should reach the same asymptotic value for a subcritical case. The Lock solution (obtained through the Hodograph method and considered "exact") [8] yields 0.335 as the value of the lift. However, Lock extends the NACA 0012 airfoil to a sharp trailing edge at $x / c=1.0089$, but does not normalize to unit length. In the present results, the NACA 0012 airfo1l is both extended and renormalized to unit length. If the Lock result is renormalized, consistent with the present results, the lift coefficient would become 0.3321 . This tends to suggest that the FP codes are in better agreement with the "exact" solution for this subcritical case.

Figure 2 is a plot of percent error in the lift coefficient versus CPU time in seconds on the Cray XMP computer for the conditions of Fig. 1 (NACA 0012, $M_{\infty}=63$, $\left.\alpha=2.0^{\circ}\right)$. The timings from all codes are based on converging the lift to an accuracy of $10^{-4}$ (four decimal digits). The time-step and convergence acceleration parameters from all codes (in general) have been set at default values; that is, a minimal amount of "tuning" has been included. Thus, the convergence rates are not optimal, but are representative of the convergence rates that would be found in practical applications. Startup times, including initialization and grid generation, 
have been subtracted from each timing. The error is computed by first constructing the asymptotic values of the lift coefficient (as done in Fig. I). Then the error is simply the absolute value of the difference between the asymptotic value and the value of the converged lift at a specific level of grid refinement. From Fig. 2 (also Fig. 1), it can be observed that the FP formulations are slightly more accurate than the Euler formulations, especially for the coarser grids. On the coarse grids, the Euler codes are more expensive than the FP codes by an average factor of about 17 , based on CPU time. For the finer grids, this factor decreases to about 11.

Figure 3 displays a plot of lift coefficient versus the average mesh spacing for a transonic case with a moderate strength shock, NACA $0012, \mathrm{M}_{\infty}=0.75$, and $\alpha=1.0^{\circ}$. No attempt was made to construct a lift error versus CPU time, as was done in Fig. 2, since, as can be seen in Fig. 3, some of the curves turn over on themselves, making the error measure potentlally misleading. We point out here that the asymptotic characteristics of both the FP and Euler formulations are grid-dependent (also apparent in Fig. 1). The algebraic and Laplacian curves for both the FP and Euler formulations show different trends and levels of accuracy. The TAIR (algebraic) and TAIR (Laplacian) results approach their limits from different directions. The level of accuracy for the Euler results is typically less for the algebralc grids, whereas the reverse is true for the FP results. The FP results all approach the same asymptotic limit to within an error of about $1 \%$. The Euler results also approach an asymptotic limit, but the error is significantly less. Another observation from Fig. 3 is that the level of accuracy owing to grid effects can be of the order of the differences in equation formulations (FP versus Euler) for these cases in which the FP is valid.

Utilizing the nonisentroplc full-potential formulation [9] in TAIR yields the middle set of curves in Fig. 3. By adding entropy effects to FP formulation, the solutions were improved to within about $4 \%$ of the Euler formulation, which it is agreed is the more valid formulation for supercritical cases.

The CPU time at convergence versus the average surface mesh spacing is plotted in Fig. 4 for the conditions shown in Fig. 3 (NACA 0012, $M_{\infty}=0.75, \alpha=1^{\circ}$ ). This yields a rough estimate of the cost of running each code for different grid sizes, without providing definitive information on the cost to obtain a desired level of accuracy. In general, the Euler codes are more expensive than the FP codes - by a factor of 10 based on CPU time and twice that based on operation count. An interesting observation is that both the ARC2D (Euler) and TAIR (FP) codes converge faster on the Laplacian grid than on the algebraic grid. In fact, the difference between TAIR (algebraic) and TAIR (Laplacian) convergence times is quite large (as much as a factor of 4). The cause for this behavior is not known for certain, but it may be that the stretching is too rapid in the algebraic grids. Because the FP formulation is based on a second-order PDE, it is more likely to be adversely affected by a grid that is nonsmooth or rapidly stretched.

Figure 5 illustrates the asymptotic $11 \mathrm{ft}$ behavior for a strong shock case ( $\operatorname{RAE} 2822, \mathrm{M}_{\infty}=0.75, \alpha=3.0^{\circ}$ ). Note that these conditions are considered to be beyond the valid range of the full-potential formulation, and only the TAIR and ARC2D codes were run for this case. The FLO codes were not used, a result of the difficulty of the case and the lack of user experience. It can be seen that the results for the TAIR code (algebralc and Laplacian grids) both reach the same asymptotic value of lift. The value obtained is about 1.69 , which is grossly in error relative to the Euler results. Thus, the FP formulation is unacceptable for this calculation. The asymptotic values for the ARC2D code (algebraic and Laplacian grids) are in good agreement producing an asymptotic value of 1 ift coefficient near 1.12 . The effect of the FP entropy correction is seen to make a major difference in the FP solution, producing errors of a level comparable to those in the previously discussed case (NACA $0012, M_{\infty}=0.75, \alpha=1^{\circ}$ ). This improvement in lift is also reflected in a comparable improvement in the surface-pressure distribution, for the nonisentropic FP pressure distribution is in good agreement with the Euler pressure distribution.

Figure 6 presents a comparison of CPU time versus grid refinement for the RAE case. Again we note about an order of magnitude difference in CPU time for FP over Euler. For this case, which is admittedly difficult for isentropic FP, the convergence rates are strongly affected by the different grids. Again, the nonisentropic formulation helped improve the convergence speed of TAIR (Laplacian). 
Figure 7 presents a plot of the convergence speed ratio (Euler to FP) versus the average surface mesh spacing for the NACA $0012, M_{\infty}=0.75, \alpha=1^{\circ}$ case. The convergence speed ratio is plotted based on two criteria: (1) CPU time, and (2) total operation count. Each data point plotted in F1g. 7 is obtained by means of a simple arithmetic average of the results for each formulation, three Euler and five FP (see F1g. 4). Although not monotonic, useful information can be obtanned from these curves. The average convergence ratio based on total operations fluctuates from about 9 to 16, and based on CPU time the fluctuation is 4 to 8 . The reason for the difference in average convergence speed ratio based on CPU time relative to total operation count is associated with vectorization efficiency. That is, the Euler codes are highly vectorized on the Cray XMP, but the FP codes are not. The Eulerto-FP speed rat10, based on CPU time, could be higher if the FP codes were more efficiently vectorized. However, the possible improvement in FP vectorization efficiency is difficult to estimate, since the AF2 algorithm in two dimensions cannot be vectorized as efficiently as the classical ADI-like implicit schemes or explicit methods. (Note that the AF2 algorithm in three dimensions does not have this disadvantage.)

In Fig. 8, an attempt is made to shed some light on an interesting controversy in which the Euler and FP formulations are involved: the proper level of solution convergence. Because of the differencing of the dependent varlable $\phi$ to obtain the pressure distribution, truncation error is added to any FP solution. Since this error adds to the lack-of-convergence error (theoretically), the FP solution must be converged more tightly than the Euler solution for the same level of accuracy in the lift calculation. Figure 8 shows a plot of error in lift versus rms error in the dependent variable ( $E_{\mathrm{rms}}$ ), pressure for the Euler formulation, and $\phi$ for the FP formulation. The exact definitions for these two different types of error are displayed 1n F1g. 8. The two curves shown in F1g. 8 were produced from the NACA 0012 , $M_{\infty}=0.75, \alpha=1^{\circ}$ case. Initially, the test case was run until tight convergence was obtained. Then, the converged dependent variables and converged lift coefficient were saved and the case was rerun. The curves shown in Fig. 8 were obtained by plotting the $11 \mathrm{ft}$ error versus the rms dependent-variable error every 50 iterations. Convergence in this case for FP and Euler solutions were about 300 and 1600 iterations, respectively. This explains the difference in number of data points plotted for each code. For this case, the FP solution does need to be converged more tightly for the same error in lift. For a lift error of about $10^{-4}$, the FP solution needs to be dropped about an order more in rms error.

\section{CONCLUSIONS}

A study involving four transonfc airfoli computer codes, two FP and two Euler, has been performed. The major conclusions of the study are as follows: (1) the FP codes are faster than the Euler codes by about an order of magnitude based on CPU t1me on the Cray XMP; (2) the FP formulation loses accuracy as transonic flow develops, but entropy corrections yleld FP solutions comparable to those of the Euler; (3) grid coarseness and type can be significant in affecting both accuracy and convergence characteristics; (4) the FP formulation must be more tightly converged than the Euler formulation for comparable levels of accuracy in the lift coefficient; and (5) in general, good accuracy for adequate meshes can be obtalned with both formulations, irrespective of the solution method.

\section{REFERENCES}

1. Holst, T. L., "A Fast, Conservative Algorithm for Solving the Transonic Ful1Potential Equation," AIAA J., Vol. 18, No. 12, Dec. 1980, pp. 1431-1439.

2. Dougherty, F. C., Holst, T. L., Gundy, K. L., and Thomas, S. D., "TAIR - A Transonic Airfoil Analysis Computer Code," NASA TM-81296, May 1981.

3. Jameson, A., "Acceleration of Transonic Potential Flow Calculations on Arbitrary Meshes by the Multiple Grid Method," ALAA Paper 79-1458, July 1979.

4. Steger, J., "Implicit Finite-Difference Simulation of Flow about Arbitrary TwoDimensional Geometries," J. Comp. Phys., Vol. 16, 1978, pp. 679-686.

5. Pulliam, T. H., "Implicit Finite-Difference Methods for the Euler Equations," Advances in Computational Transonics, Ed. W. Habashi, Pineridge Press Ltd., Swansea, U.K., 1983. 
6. Jameson, A., Schmidt, W., and Turke1, R., "Numerical Solutions of the Euler Equations by Finite-Volume Methods Using Runge-Kutta Time-Stepping Schemes," AIAA Paper 81-1259, June 1981.

7. Pulliam, T. H., Jespersen, D. C., and Childs, R. E., "An Enhanced Version of an Implic1t Code for the Euler Equations," AIAA Paper 83-0344, Jan. 1981.

8. Lock, R. C., "Test Cases for Numerical Methods in Two-Dimensional Transonic Flows," AGARD Report No. 575, 1970.

9. Hafez, M. and Lovell, D., "Entropy and Vorticity Corrections for Transonic Flows," AIAA Paper 83-1926, July 1983.
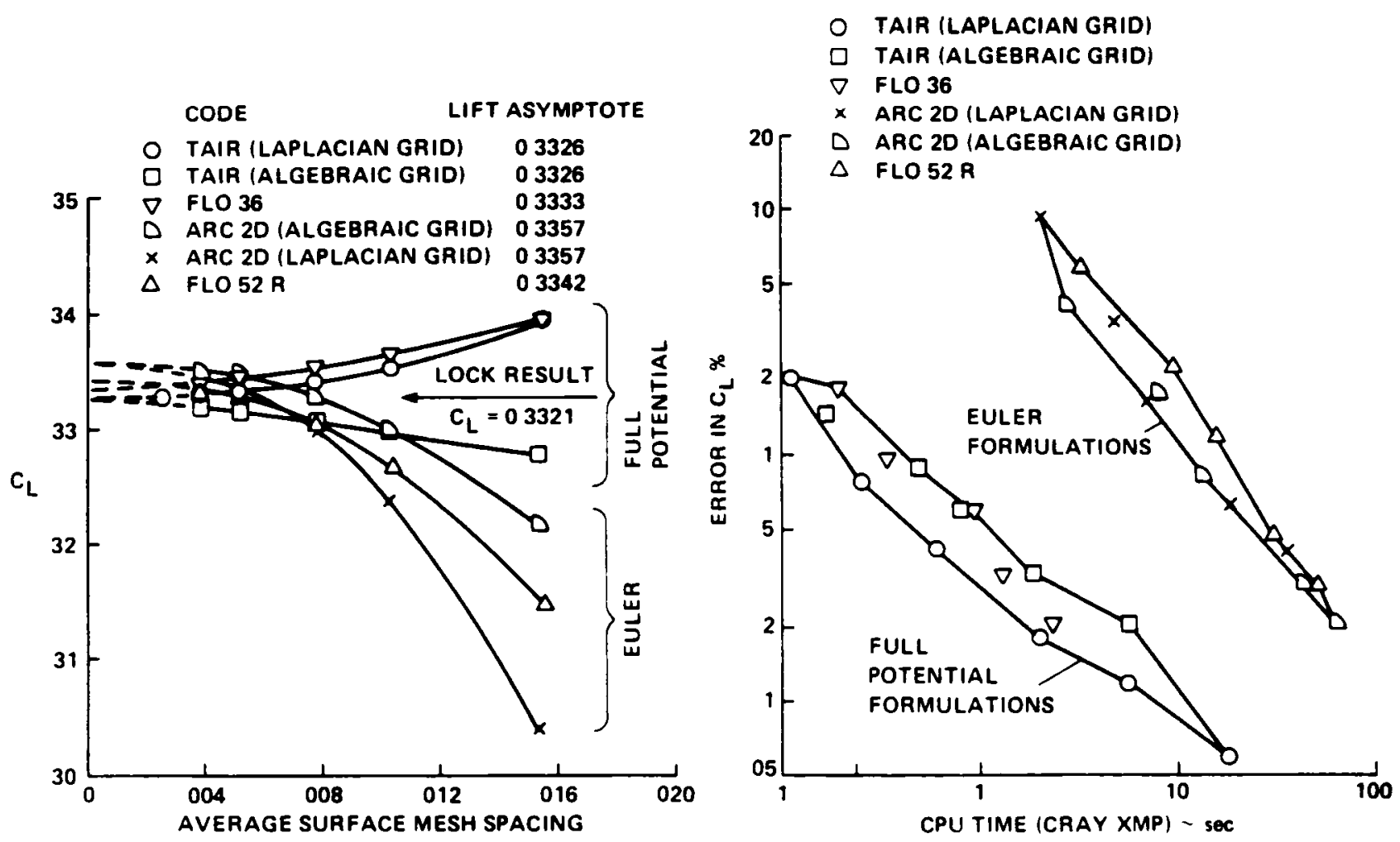

Fig. 1 Lift versus grid refinement: NACA $0012, M_{\infty}=0.63, \alpha=2^{\circ}$.

F1g. 2 Lift error versus CPU: NACA 0012 , $M_{\infty}=0.63, \alpha=2^{\circ}$. 


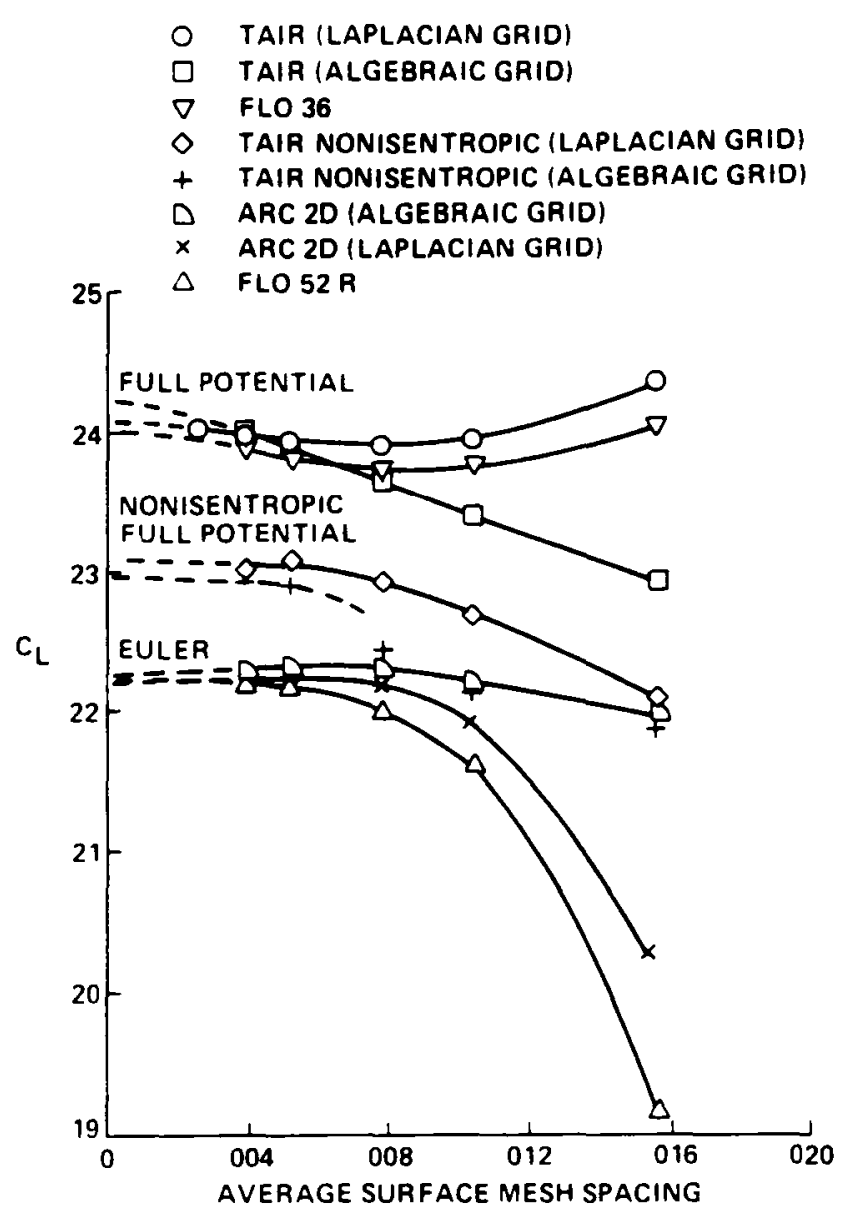

F1g. 3 Lift versus grid refinement: NACA 0012, $M_{\infty}=0.75, \alpha=1^{\circ}$.
O TAIR (LAPLACIAN GRID)

- TAIR (ALGEBRAIC GRID)

$\nabla$ FLO 36

- TAIR NONISENTROPIC (LAPLACIAN GRID)

+ TAIR NONISENTROPIC (ALGEBRAIC GRID)

$\triangle$ FLO 52 R

D ARC 2D (ALgEbRAIC GRID)

$\times$ ARC $2 D$ (LAPLACIAN GRID)

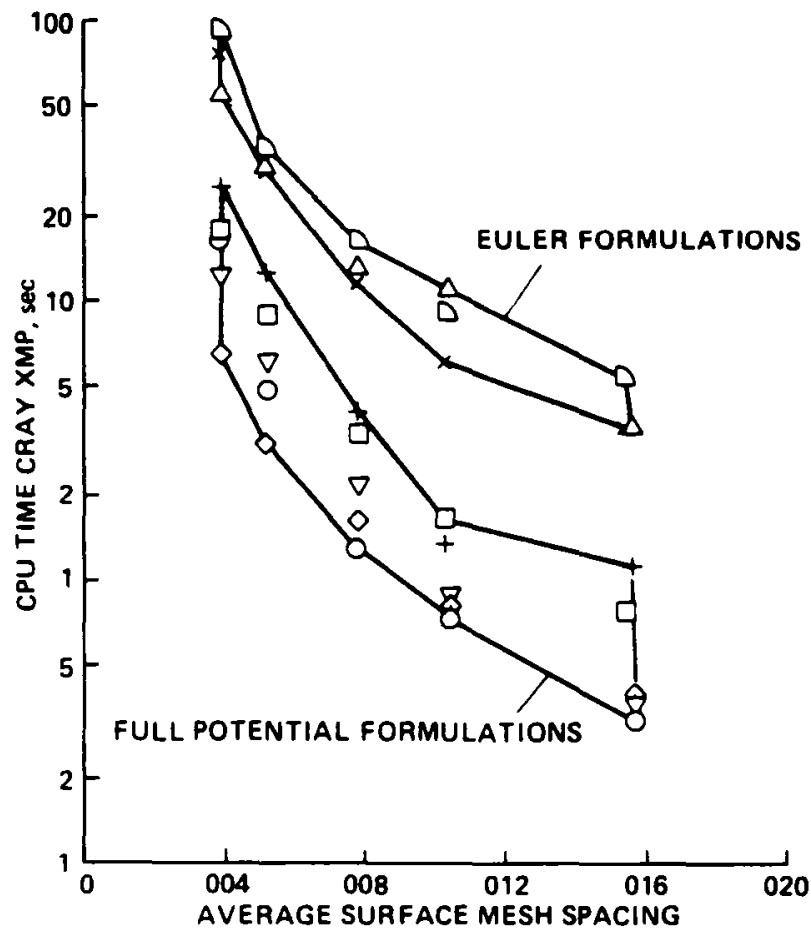

F1g. 4 CPU versus grid refinement: NACA $0012, M_{\infty}=0.75, \alpha=1^{\circ}$.

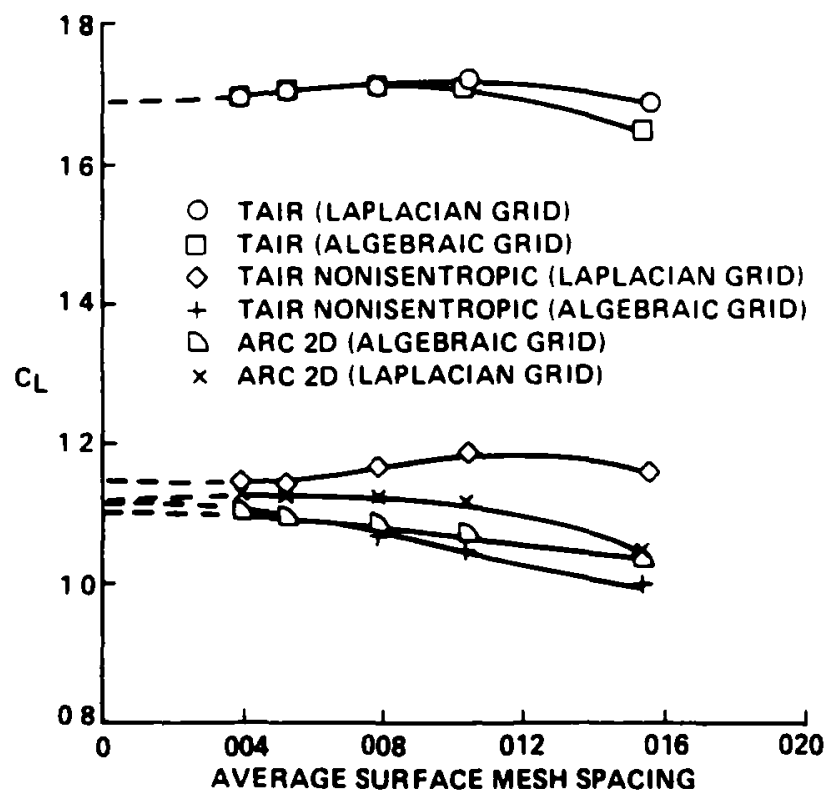

Fig. 5 Lift versus grid refinement: $\operatorname{RAE} 2822, M_{\infty}=0.75, a=3^{\circ}$. 


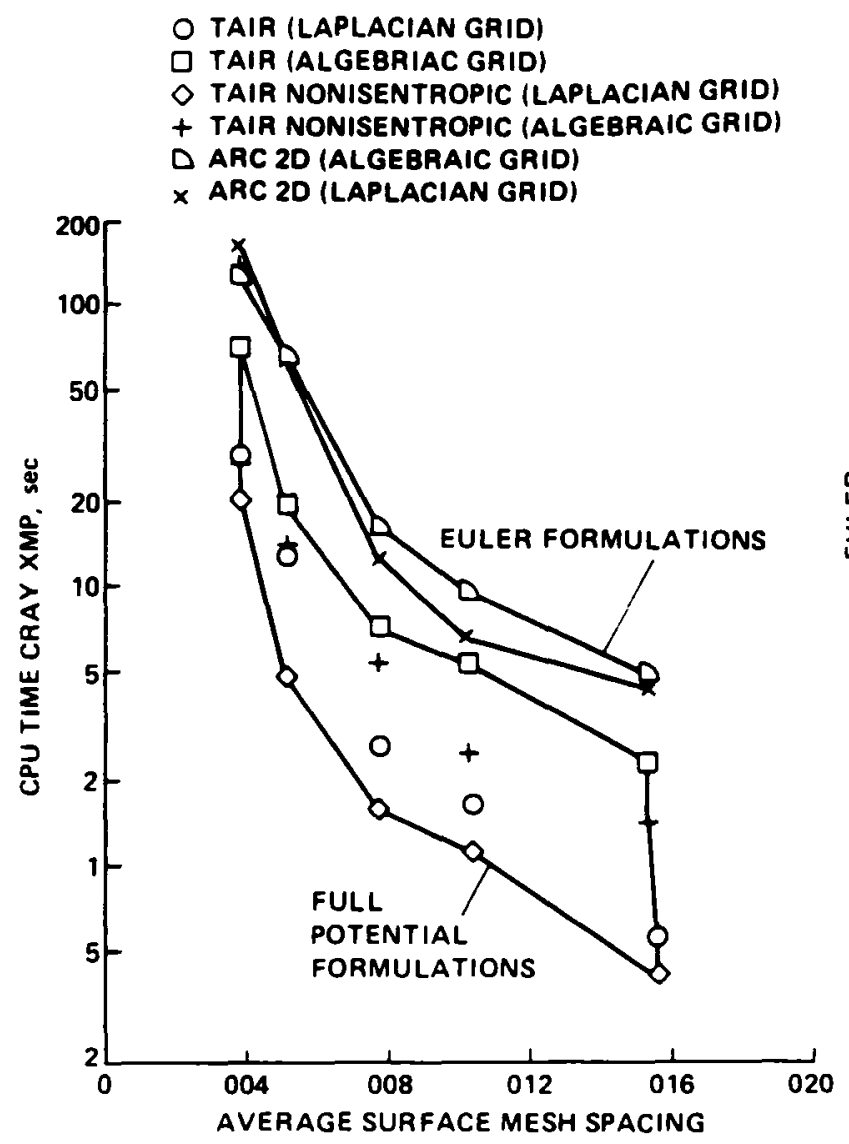

F1g. 6 CPU versus grid refinement: $\operatorname{RAE} 2822, M_{\infty}=0.75, \alpha=3^{\circ}$.

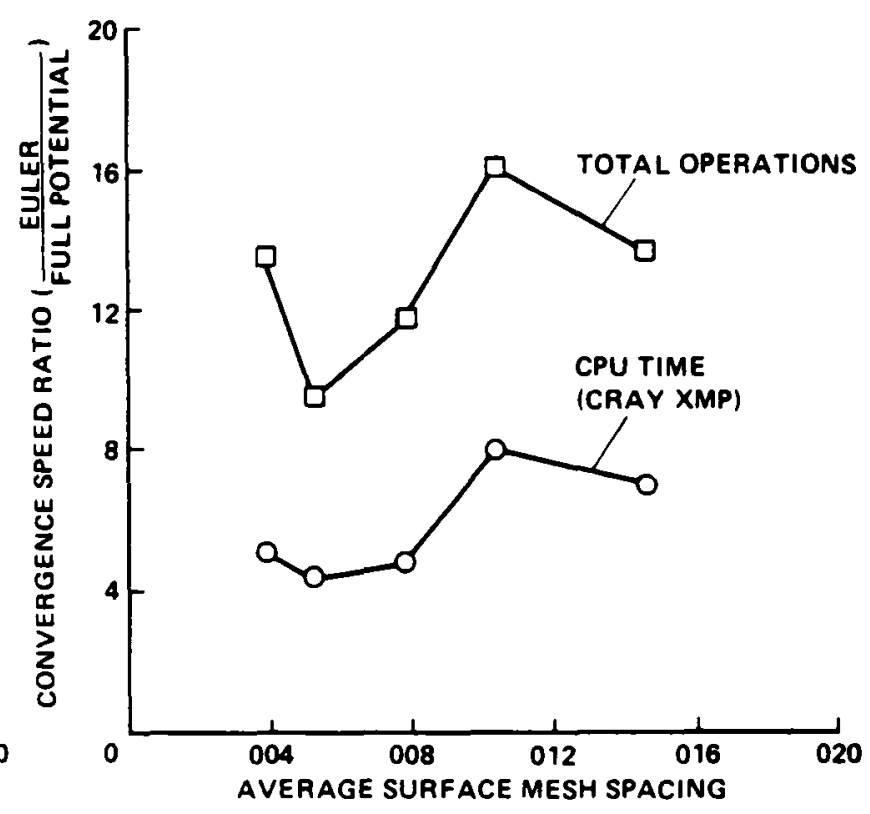

Fig. 7 Convergence speed versus grid refinement: NACA $0012, \mathrm{M}_{\infty}=0.75$, $\alpha=1^{\circ}$.

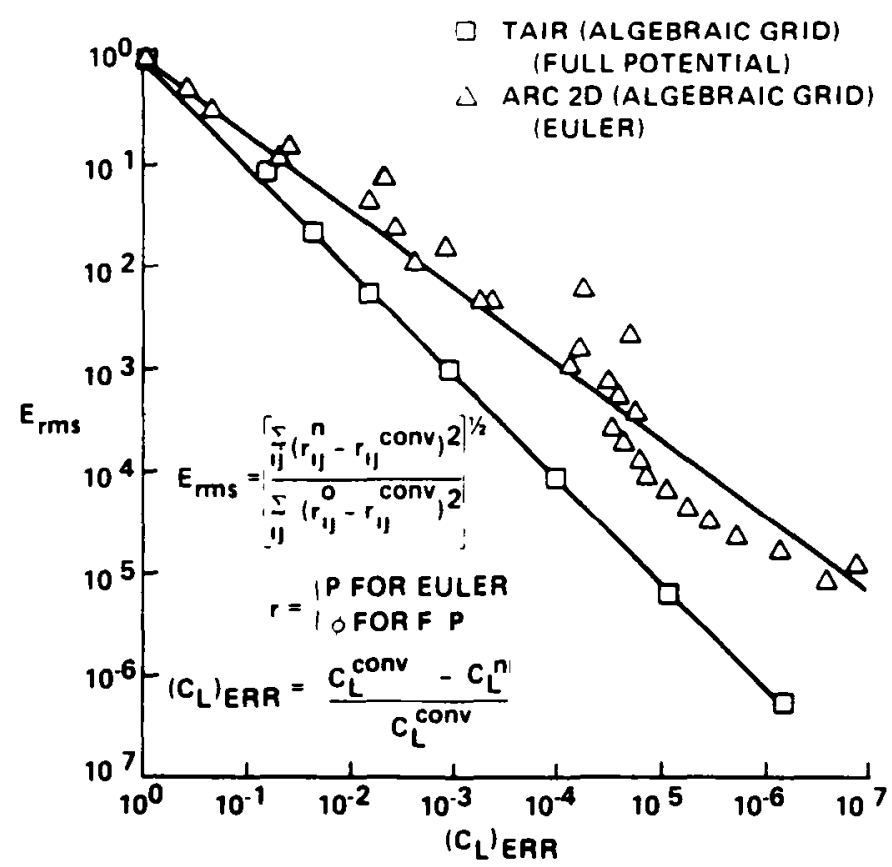

Fig. 8 Root-mean-square error versus lift error: NACA 0012, $M_{\infty}=0.75, \alpha=1^{\circ}$. 


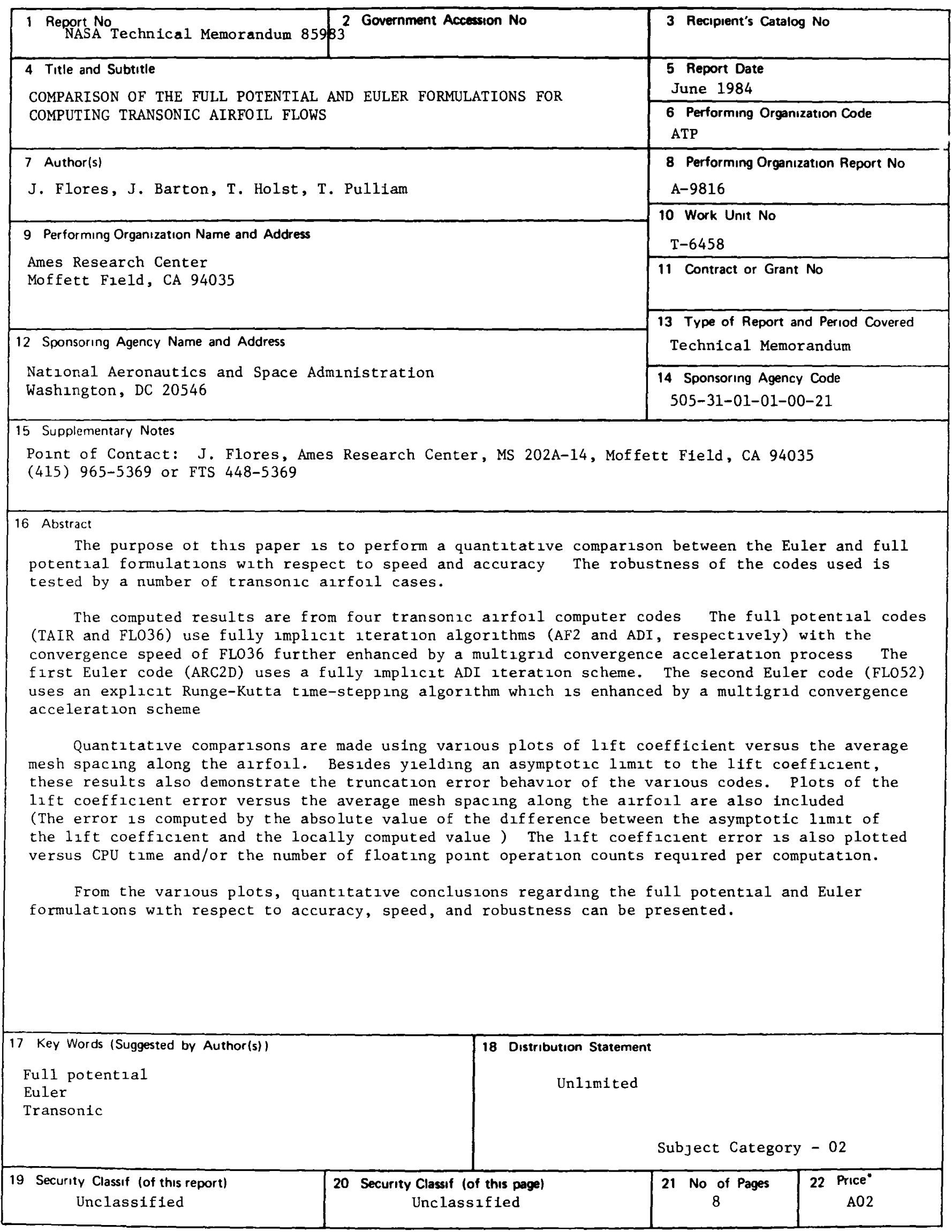


End of Document 